\title{
The evolution of free wave packets
}

\author{
Mark Andrews ${ }^{\text {a) }}$ \\ Department of Physics, Australian National University, ACT 0200, Australia
}

(Received 30 December 2007; revised manuscript received 26 August 2008)

\begin{abstract}
We discuss four general features of the force-free evolution of wave packets: (1) The spatial spread of a packet changes with time in a simple way. (2) For sufficiently short durations (related to the spread in the momentum of the packet) the probability distribution will move with uniform speed and little change in shape. (3) After a sufficiently long time (related to the initial spatial spread) the wave function converges to a simple form that is simply related to the momentum distribution of the packet. In this asymptotic regime the shape of the probability distribution no longer changes, and its scale increases linearly with the time. (4) There is an infinite denumerable set of simple wave packets (the Hermite-Gauss packets) that do not change shape at any time during their evolution. () 2008 American Association of Physics Teachers.
\end{abstract}

[DOI: 10.1119/1.2982628]

\section{INTRODUCTION}

The behavior of free wave packets as they evolve in time is striking to many students of quantum mechanics. A wave packet usually changes shape and always eventually spreads out without limit. How different this behavior is from that of a free classical particle. The reconciliation of the classical and quantum descriptions is part of the bigger question of the interpretation of quantum mechanics; here we will consider only the dynamics of free wave packets as given by Schrödinger's equation.

We will consider only one-dimensional systems, but the results apply to each dimension of a two or threedimensional system because the Hamiltonian $\hat{H}=\hat{\mathbf{p}}^{2} / 2 m$ in Cartesian coordinates is a sum of one-dimensional Hamiltonians. (We will consistently use a hat to indicate an operator.) Systems with more than one dimension can display a greater richness in behavior, because the parameters of the wave function may be different in each dimension.

Most texts derive the propagator ${ }^{1}$

$$
K\left(x, x^{\prime}, t\right)=\sqrt{m / 2 \pi i \hbar t} \exp \left[i m\left(x-x^{\prime}\right)^{2} / 2 \hbar t\right]
$$

such that the evolution of an arbitrary initial wave packet $\psi(x, 0)$ is

$$
\psi(x, t)=\int_{-\infty}^{\infty} K\left(x, x^{\prime}, t\right) \psi\left(x^{\prime}, 0\right) d x^{\prime}
$$

There are not many wave packets for which this integral can be easily evaluated, and the only case commonly treated is the Gaussian packet. Therefore, the evolution of free wave packets remains obscure to many students.

Ehrenfest's result that $\langle\hat{p}\rangle$ is constant and $\langle\hat{x}\rangle=\langle\hat{x}\rangle_{0}$ $+\langle\hat{p}\rangle t / m$ is well known. (Angled brackets will be used to indicate expectation values; thus, $\langle\hat{a}\rangle$ represents the expectation value of the operator $\hat{a}$.) There are four other general results on the free evolution of wave packets ${ }^{2}$ that can be simply derived and greatly improve the general understanding of this evolution.
(1) The spread of any wave packet as measured by $\Delta_{x}$ $=\left\langle(\hat{x}-\langle\hat{x}\rangle)^{2}\right\rangle^{1 / 2}$ increases in time as

$$
\Delta_{x}=\sqrt{\Delta_{\min }^{2}+\left(t-t_{\min }\right)^{2} \Delta_{p}^{2} / m^{2}},
$$

where $\Delta_{\min }$ is the minimum value of the spread (taken at time $\left.t_{\min }\right)$ and $\Delta_{p}=\left\langle(\hat{p}-\langle\hat{p}\rangle)^{2}\right\rangle^{1 / 2}$ does not change with time. If the wave function is real everywhere (which can be true only for an instant), the packet has its minimum spread $\Delta_{\min }$ at that instant.

(2) For time intervals much less than $m \hbar / \Delta_{p}^{2}$, the wave packet moves with speed $\langle\hat{p}\rangle / m$ with little change in the shape of the probability distribution.

(3) The asymptotic evolution for times $|t| \gg m \Delta_{x}^{2} / \hbar$ converges to a functional form simply related to the momentum distribution of the packet, given by the Fourier transform of the initial wave function. There are many forms of initial wave packet for which the asymptotic evolution can be easily determined, because there are many functions for which the Fourier transform can be simply evaluated. These two time regimes cannot overlap because of Heisenberg's uncertainty relation, $\Delta_{x} \Delta_{p} \geqslant \hbar / 2$.

(4) There is an infinite number of simple wave packets that do not change shape as they evolve. These wave functions have the form of a Gaussian multiplied by an Hermite polynomial in $x$. By shape we mean the functional form of the probability distribution; the scale (and consequently the normalization) may change with time. Thus, a wave function $\psi(x, t)$ such that $|\psi(x, t)|^{2}=\gamma(t)^{-1} f(x / \gamma(t))$ does not change shape. [The factor $\gamma(t)^{-1}$ is required to preserve normalization.] All wave packets evolve to an asymptotic form whose shape does not change.

The simplest of the wave packets that do not change shape as they evolve is the real Gaussian, which evolves to become complex but retains its Gaussian shape. A recent publication ${ }^{3}$ claims that all wave packets become "approximately Gaussian" after a long enough time. This claim is too broad; there are many wave packets that will never become anything like a Gaussian. For example, any antisymmetric wave function will remain antisymmetric. Furthermore, the Hermite-Gauss wave packets retain their shape as they evolve and, apart from the pure Gaussian, none of them could be described as "approximately Gaussian." 


\section{THE EVOLUTION OVER SHORT PERIODS}

We start with the well-known expression for the evolution of a wave packet in terms of the initial momentum distribution $\phi(p){ }^{4}$

$$
\psi(x, t)=\frac{1}{\sqrt{2 \pi \hbar}} \int_{-\infty}^{\infty} \exp \left[\frac{i}{\hbar}\left(p x-\frac{1}{2 m} p^{2} t\right)\right] \phi(p) d p,
$$

and express $p^{2}$ in Eq. (4) in terms of $p-\bar{p}$ as $p^{2}=\bar{p}^{2}+2 \bar{p}(p$ $-\bar{p})+(p-\bar{p})^{2}$, where $\bar{p}=\langle\hat{p}\rangle{ }^{5}$ We expect $\phi(p)$ to be small if $(p-\bar{p})^{2} \gg \Delta_{p}^{2}$. Therefore, if $\Delta_{p}^{2} t / 2 m \hbar \ll 1$, the term $(p-\bar{p})^{2}$ will make a negligible contribution to the phase of the exponential. In other words, the integrand contains a factor $\exp \left[i(p-\bar{p})^{2} t / 2 m \hbar\right]$ that is close to unity throughout the range of $p$ where $\phi(p)$ has any significant magnitude. Hence,

$$
\begin{aligned}
\psi(x, t) \approx & \frac{1}{\sqrt{2 \pi \hbar}} \exp \left(\frac{i \bar{p}^{2} t}{2 m \hbar}\right) \\
& \times \int_{-\infty}^{\infty} \exp \left[\frac{i}{\hbar} p\left(x-\frac{\bar{p} t}{m}\right)\right] \phi(p) d p \\
= & \exp \left(\frac{i \bar{p}^{2} t}{2 m \hbar}\right) \psi\left(x-\frac{\bar{p} t}{m}, 0\right) .
\end{aligned}
$$

The wave function moves with speed $\bar{p} / m$ and no change in shape, just a change in phase. A more rigorous approach to the error in this approximation is given in Sec. VII.

We have taken the initial time to be $t=0$, but this analysis can be applied at any time. Because $\Delta_{p}$ does not change, the time scale $m \hbar / 2 \Delta_{p}^{2}$ for changes in the shape applies throughout the evolution.

If the wave function has any discontinuity, then $\Delta_{p}=\infty$ and there will be very rapid changes. An example of this behavior will be considered in Sec. VI.

\section{THE EVOLUTION FOR LARGE TIMES}

For the propagator in Eq. (1) we write the term $\left(x-x^{\prime}\right)^{2}$ in the exponent as $x^{2}-\bar{x}^{2}-2(x-\bar{x}) x^{\prime}+\left(x^{\prime}-\bar{x}\right)^{2}$, with $\bar{x}=\langle\hat{x}\rangle_{t=0}$. We expect $\psi\left(x^{\prime}, 0\right)$ to be small if $\left(x^{\prime}-\bar{x}\right)^{2} \gg \Delta_{x}^{2}$ and therefore for all times (future and past) with $|t| \gg m \Delta_{x}^{2} / \hbar$, the term in $\left(x^{\prime}-\bar{x}\right)^{2}$ will make a negligible change to the phase of the exponential. Hence

$$
\begin{aligned}
\psi(x, t) \approx & \sqrt{\frac{m}{2 \pi i \hbar t}} \exp \left[\frac{i m}{2 \hbar t}\left(x^{2}-\bar{x}^{2}\right)\right] \\
& \times \int_{-\infty}^{\infty} \exp \left[-\frac{i m}{\hbar t}(x-\bar{x}) x^{\prime}\right] \psi\left(x^{\prime}, 0\right) d x^{\prime} \\
= & \sqrt{\frac{m}{i t}} \exp \left[\frac{i m}{2 \hbar t}\left(x^{2}-\bar{x}^{2}\right)\right] \phi\left(\frac{m}{t}(x-\bar{x})\right),
\end{aligned}
$$

where

$$
\phi(p)=\frac{1}{\sqrt{2 \pi \hbar}} \int_{-\infty}^{\infty} \exp \left(-i p x^{\prime} / \hbar\right) \psi\left(x^{\prime}, 0\right) d x^{\prime},
$$

which is the initial momentum wave function of the packet. A more rigorous derivation of the error in this approximation is given in Sec. VII. This asymptotic form is important in the theory of scattering. ${ }^{6}$

The asymptotic probability distribution is therefore given by $(m / t)|\phi(m(x-\bar{x}) / t)|^{2}$. This result has a simple interpretation: $m(x-\bar{x}) / t$ is the momentum required for the particle to be at position $x$ at time $t$ if it was at $\bar{x}$ at time $t=0$. So, the probability of finding the particle at $x$ is proportional to the probability that it had the right momentum to get there (ignoring the fact that the initial distribution was spread over a distance of order $\Delta_{x}$ about $\bar{x}$ ).

Let us introduce the times $t_{p}=m \hbar / 2 \Delta_{p}^{2}$ and $t_{x}=2 m \Delta_{x}^{2} / \hbar$. Then $t_{p} \leqslant t_{x}$ (from the uncertainty relation) and their equality implies a Gaussian packet. In general, $t_{p}$ gives the time scale for changes in shape (including the changes in spatial scale) while $t_{x}$ gives the time scale to reach the asymptotic regime where there is no further change in shape and $|\psi|$ expands uniformly with time.

Problem 1. For the initial triangular wave packet with

$$
\psi(x, 0)= \begin{cases}a-|x| & \text { for }|x| \leqslant a \\ 0 & \text { otherwise }\end{cases}
$$

show that the asymptotic behavior for large $|t|$ is such that $|\psi(x, t)| \propto t^{3 / 2} x^{-2} \sin ^{2}(x m a / 2 \hbar t)$, and the time scale beyond which this behavior will be approached is $m a^{2} / \hbar$.

\section{THE EVOLUTION OF THE SPREAD $\Delta_{x}$}

To find how $\left\langle(\hat{x}-\langle\hat{x}\rangle)^{2}\right\rangle$ varies with time we use the standard result that for any operator $\hat{A}(t)$

$$
d_{t}\langle\hat{A}(t)\rangle=\left\langle\partial_{t} \hat{A}(t)\right\rangle+(i / \hbar)\langle[\hat{H}, \hat{A}(t)]\rangle,
$$

where $\hat{H}$ is the Hamiltonian operator. (We use $d_{t}$ to denote the time derivative $d / d t$ and $\partial_{t}$ for the partial derivative $\partial / \partial t$. In this case $\partial_{t} \hat{A}(t)$ will apply only to any explicit dependence of $\hat{A}(t)$ on the time; that is, $\hat{x}$ and $\hat{p}$ are regarded as being independent of the time when taking the partial derivative.) The calculation is somewhat tedious and is given as a problem in some textbooks. ${ }^{7,8}$ It becomes much simpler if we introduce the shorthand notation

$$
D \hat{A}(t) \equiv \partial_{t} \hat{A}(t)+(i / \hbar)[\hat{H}, \hat{A}(t)],
$$

from which it follows that $d_{t}\langle\hat{A}(t)\rangle=\langle D \hat{A}(t)\rangle$, and

$$
D(\hat{A}(t) \hat{B}(t))=\hat{A}(t) D \hat{B}(t)+(D \hat{A}(t)) \hat{B}(t) .
$$

For a free particle $D \hat{x}=\hat{p} / m$ and $D \hat{p}=0$. We introduce the time-dependent operators $\hat{X}=\hat{x}-\langle\hat{x}\rangle$ and $\hat{P}=\hat{p}-\langle\hat{p}\rangle$. It follows that $D \hat{X}=\hat{P} / m$ and $D \hat{P}=0$. Then, using Eq. (11), we have $D \hat{X}^{2}=2 \hat{R} / m$, where $\hat{R}=(\hat{P} \hat{X}+\hat{X} \hat{P}) / 2$, and $D \hat{R}=\hat{P}^{2} / m$. Thus, $d_{t}\langle\hat{R}\rangle=\left\langle\hat{P}^{2}\right\rangle / m$ and, because $\left\langle\hat{P}^{2}\right\rangle$ is constant, it follows that

$$
m\langle\hat{R}\rangle=\left\langle\hat{P}^{2}\right\rangle\left(t-t_{0}\right),
$$

where $t_{0}$ is a constant. Thus $\langle\hat{R}\rangle$ will become zero at time $t$ $=t_{0}$. We can integrate $d_{t}\left\langle\hat{X}^{2}\right\rangle=2\langle\hat{R}\rangle / \mathrm{m}$ to give

$$
\left\langle\hat{X}^{2}\right\rangle=m^{-2}\left\langle\hat{P}^{2}\right\rangle\left(t-t_{0}\right)^{2}+\left\langle\hat{X}^{2}\right\rangle_{0},
$$

which is equivalent to Eq. (3). Note that Eq. (12) implies that, at any time $t$, we can calculate the time of minimum 
spread from $\left\langle\hat{P}^{2}\right\rangle$ and the value of $\langle\hat{R}\rangle$ at time $t$. Every wave packet has its minimum spread when $\langle\hat{R}\rangle=0$.

If the wave function $\psi$ is real at some time, the $i$ in Schrödinger's equation $\partial_{t} \psi=-i(\hbar / 2 m) \partial_{x}^{2} \psi$ shows that $\psi$ will immediately become complex. Also, $\langle\hat{p}\rangle=0$ because $\langle\hat{p}\rangle$ $=\int \psi \hat{p} \psi d x=\int(\hat{p} \psi)^{*} \psi d x=-\int(\hat{p} \psi) \psi d x=-\langle\hat{p}\rangle$. Similarly, $\langle\hat{p} \hat{x}\rangle$ $=\int \psi \hat{p} \hat{x} \psi d x=\int(\hat{p} \psi)^{*} \hat{x} \psi d x=-\int(\hat{p} \psi)(\hat{x} \psi) d x=-\langle\hat{x} \hat{p}\rangle$. That is, $\langle\hat{R}\rangle=0$ and therefore the wave packet has its minimum spread at that instant. This result also shows that a free wave packet which is real at some time cannot be real at any other time. In fact, the probability is symmetric in time about the instant when the wave function was real, because if $\psi(x, t)$ satisfies Schrödinger's equation, then so does $\psi^{*}\left(x, 2 t_{0}-t\right)$. Because these two functions are equal at $t=t_{0}$ if $\psi\left(x, t_{0}\right)$ is real, they must be equal at all times.

Given a wave packet $\xi$ that is at rest (in the sense that $\langle\hat{p}\rangle_{\xi}=0$ and therefore $\langle\hat{x}\rangle_{\xi}$ is constant), we can make a moving packet by multiplying the wave function by $\exp (i p x / \hbar)$. With $\psi\left(x, t_{0}\right)=\exp (i p x / \hbar) \xi\left(x, t_{0}\right)$, it follows that $\hat{p} \psi=p \psi$ $+\exp (i p x / \hbar) \hat{p} \xi$, and therefore $\langle\hat{p}\rangle_{\psi}=p$. Similarly, $\left\langle\hat{p}^{2}\right\rangle_{\psi}=p^{2}$ $+\left\langle\hat{p}^{2}\right\rangle_{\xi}$, and hence $\left(\Delta_{p}\right)_{\psi}=\left(\Delta_{p}\right)_{\xi}$. Also, $\hat{p}(x \psi)=p x \psi$ $+\exp (i p x / \hbar) \hat{p}(x \xi)$, and therefore $\langle\hat{p} \hat{x}+\hat{x} \hat{p}\rangle_{\psi}=2 p\langle\hat{x}\rangle_{\xi}+\langle\hat{p} \hat{x}$ $+\hat{x} \hat{p}\rangle_{\xi}$. Thus, $\langle\hat{R}\rangle_{\psi}=\langle\hat{R}\rangle_{\xi}$, and it follows that the evolution of the spatial spread is the same as for $\xi$.

The evolution of $\psi$ can be obtained from that of $\xi$ by applying a Galilean transformation, ${ }^{9}$

$$
\psi(x, t)=\exp \left(\frac{i}{\hbar}\left(p x-p^{2} \frac{t-t_{0}}{2 m}\right)\right) \xi\left(x-p \frac{t-t_{0}}{m}, t\right) .
$$

It is easy to check that this form of $\psi$ satisfies Schrödinger's equation if $\xi$ does and that $\psi\left(x, t_{0}\right)=\exp (i p x / \hbar) \xi\left(x, t_{0}\right)$.

A Gaussian packet (that is, a wave function of the form $\exp \left[a x^{2}+b x+c\right]$, where $a, b$, and $c$ may be complex) will remain Gaussian as it evolves, as can be seen by inserting a Gaussian into the propagation integral in Eq. (1). A real Gaussian wave packet can be written as $\psi(x)$ $=\exp \left(-x^{2} / 2 d^{2}\right)$, apart from normalization and a shift in origin. Then $\left\langle x^{2}\right\rangle=d^{2} / 2,\left\langle p^{2}\right\rangle=\hbar^{2} / 2 d^{2}$ and the uncertainty product $\Delta_{x} \Delta_{p}$ is $\hbar / 2$, which shows that every real Gaussian is a minimum uncertainty state. A packet can have the minimum uncertainty property only for an instant, because every packet evolves with $\Delta_{p}$ fixed and $\Delta_{x}$ changing. It was shown above that a Gaussian of the form $\exp \left(i p x / \hbar-x^{2} / 2 d^{2}\right)$ has the same $\Delta_{x}$ and $\Delta_{p}$ as when $p=0$ and therefore is also a minimum uncertainty state. It is shown in many texts ${ }^{10}$ that every minimum uncertainty state has this form.

Problem 2. The initial wave packet $\psi(x)$ $=\cos (p x / \hbar) \exp \left(-x^{2} / 2 d^{2}\right)$ can be expressed as the superposition of the two packets $\exp \left(i p x / \hbar-x^{2} / 2 d^{2}\right)$ and $\exp \left(-i p x / \hbar-x^{2} / 2 d^{2}\right)$. Apply the results of the previous paragraph and Eq. (13) to each of these two packets to show that if $p \gg \hbar / d$, then $\psi(x)$ will evolve into two separate packets, and if $p \ll \hbar / d$, the two packets will overlap indefinitely. [Use the values of $\left\langle x^{2}\right\rangle$ and $\left\langle p^{2}\right\rangle$ for the real Gaussian given in the previous paragraph. It is not necessary to evaluate any other integrals or to calculate the explicit time evolution of any wave functions.]

Problem 3. (a) Show that the minimum spread $\Delta_{\text {min }}$ of any wave packet is given by $\Delta_{\min }^{2}=\left(\left\langle X^{2}\right\rangle\left\langle P^{2}\right\rangle-\langle R\rangle^{2}\right) /\left\langle P^{2}\right\rangle$. (b) For the general Gaussian packet $\psi(x)=\exp \left(a x^{2}+b x+c\right)$, where $a$, $b$, and $c$ may be complex, it is straightforward to show that $\left\langle X^{2}\right\rangle=1 / 4 \alpha,\left\langle P^{2}\right\rangle=\left(\alpha^{2}+\beta^{2}\right) / \alpha$, and $\langle R\rangle=\beta / 2 \alpha$, where $a=$ $-\alpha+i \beta$. Use these results to show that every Gaussian packet becomes a minimum uncertainty packet at some time.

Problem 4. Consider a wave packet $\psi(x)$ that is initially real and symmetric. Then, its momentum wave function $\phi(p)$ is also real and symmetric. From Eq. (6) $\chi(x, t)=\sqrt{m / t} \phi((x$ $-\bar{x}) m / t)$ is real and, for large times, has the same magnitude as the complex evolution $\psi(x, t)$ of $\psi(x)$. That is, $\chi(x, t)$ is an envelope to the real and imaginary parts of $\psi(x, t)$. Although $\chi(x, t)$ will have the same spatial spread as $\psi(x, t)$, the spread in momentum will be very different. Show that

$$
\begin{aligned}
& \left\langle(\hat{x}-\bar{x})^{2}\right\rangle_{\chi}=(t / m)^{2}\left\langle(\hat{p}-\bar{p})^{2}\right\rangle_{\psi(x)} \\
& \left\langle(\hat{p}-\bar{p})^{2}\right\rangle_{\chi}=(m / t)^{2}\left\langle(\hat{x}-\bar{x})^{2}\right\rangle_{\psi(x)} .
\end{aligned}
$$

It follows that the uncertainty product $\Delta_{x} \Delta_{p}$ of the envelope of the completely evolved packet will be the same as that of the initial packet.

\section{THE HERMITE-GAUSS WAVE PACKETS}

We will first find the most general solution of the free Schrödinger equation that is of Gaussian form

$$
\Psi(x, t)=\exp \left[a(t) x^{2}+b(t) x+c(t)\right] .
$$

This form will enable us to generate a sequence of solutions that are Hermite polynomials multiplied by Gaussians. It is easy to verify that $\Psi(x, t)$ will be a solution if

$$
\begin{aligned}
& \dot{a}=\frac{2 i \hbar}{m} a^{2}, \\
& \dot{b}=\frac{2 i \hbar}{m} a b, \\
& \dot{c}=\frac{i \hbar}{2 m}\left(2 a+b^{2}\right) .
\end{aligned}
$$

Equation (18a) gives $a=i m / 2 \hbar\left(t+t_{c}\right)$, where $t_{c}$ is a constant that could be complex. The real part of $t_{c}$ would merely change the origin of $t$, so we take $t_{c}=-i \tau$ where $\tau$ is real, and take $\tau$ positive to make $|\Psi(x, t)|^{2}$ normalizable. Then, Eq. (18b) becomes $\dot{b}=-b /(t-i \tau)$ which gives $b=b_{0} /(t-i \tau)$. We take $b_{0}=2 \lambda \tau / d$, where we have introduced the length $d$ $=\sqrt{\hbar \tau / m}$, so that $\lambda$ is a dimensionless constant. Then, it follows that $c=-\frac{1}{2} \log (t-i \tau)-2 i \tau \lambda^{2} /(t-i \tau)+c_{0}$, where $c_{0}$ is constant. Thus,

$$
\Psi(x, t)=\frac{1}{\sqrt{t-i \tau}} \exp \left(\frac{i x^{2} / 2 d^{2}+2 \lambda x / d-2 i \lambda^{2}}{(t-i \tau) / \tau}+c_{0}\right) .
$$

[This solution of the free Schrödinger equation is essentially the same as the propagator $K\left(x, x^{\prime}, t\right)$ in Eq. (1), which is a solution except at $t=0$, where it is singular. To obtain $\Psi$ from $K$ replace $x^{\prime}$ by $2 i \lambda d$ and $t$ by $t-i \tau$, which avoids the singularity.] 
Now, we expand the solution in Eq. (19) in powers of $\lambda$ : $\Psi(x, t)=\sum_{n=0}^{\infty} \psi_{n}(x, t) \lambda^{n} / n$ !. This expansion can be achieved using the generating function for the Hermite polynomials,

$$
\exp \left(2 z s-s^{2}\right)=\sum_{n=0}^{\infty} H_{n}(z) \frac{s^{n}}{n !}
$$

If we apply Eq. (20) directly to Eq. (19) with $s$ $=\lambda \sqrt{2 i \tau /(t-i \tau)}$, we would find a complex value for the argument $z$ of $H_{n}(z)$. However, if we take $c=-\lambda^{2}$, then $z$ will be real and

$$
\Psi(x, t)=\frac{1}{\sqrt{t-i \tau}} \exp \left(\frac{i \tau x^{2}}{2 d^{2}(t-i \tau)}+\frac{2 x}{\gamma} s-s^{2}\right),
$$

where $s=\sqrt{(t+i \tau) /(t-i \tau)} \lambda$ and $\gamma=d \sqrt{1+t^{2} / \tau^{2}}$. Because $\Psi(x, t)$ must be a solution for all values of $\lambda$, it follows that for each $n$

$$
\psi_{n}(x, t)=\frac{1}{\sqrt{t-i \tau}}\left(\frac{t+i \tau}{t-i \tau}\right)^{n / 2} \exp \left(\frac{i t x^{2}}{2 \tau \gamma^{2}}-\frac{x^{2}}{2 \gamma^{2}}\right) H_{n}(x / \gamma)
$$

is a solution of the free Schrödinger equation. These Hermite-Gauss wave packets do not change their shape as they evolve, but the scale $\gamma$ does change.

The normalized probability distributions of the first few are

$$
\begin{aligned}
& \left|\psi_{0}(x, t)\right|^{2}=\frac{1}{\gamma \sqrt{\pi}} \exp \left(-x^{2} / \gamma^{2}\right) \\
& \left|\psi_{1}(x, t)\right|^{2}=\frac{2}{\gamma \sqrt{\pi} \frac{x^{2}}{\gamma^{2}}} \exp \left(-x^{2} / \gamma^{2}\right), \\
& \left|\psi_{2}(x, t)\right|^{2}=\frac{1}{2 \gamma \sqrt{\pi}}\left(1-2 x^{2} / \gamma^{2}\right)^{2} \exp \left(-x^{2} / \gamma^{2}\right)
\end{aligned}
$$

These probability distributions have the same spatial form as the energy eigenfunctions of the harmonic oscillator.

It follows from the completeness of the harmonic oscillator eigenfunctions that this set of solutions forms a complete basis for the expansion of an arbitrary wave function at any particular time. This result provides an alternative method of calculating the free evolution of any wave function

Each of the solutions $\psi_{n}(x, t)$ remains centered on the origin. To obtain the solutions that move uniformly with speed $p / m$, we can either multiply by $\exp (i p x / \hbar)$ and carry out the Galilean transformation in Eq. (14) or we can modify the generating solution by replacing $\lambda$ by $\lambda+p d / 2 \hbar$, so that

$$
\begin{aligned}
\Psi(x, t)= & \frac{1}{\sqrt{t-i \tau}} \exp \left[\frac{i}{\hbar}\left(p x-\frac{p^{2} t}{2 m}\right)\right] \\
& \times \exp \left[i \frac{\tau(x-p t / m)^{2}}{2 d^{2}(t-i \tau)}+2 \lambda \frac{\tau(x-p t / m)}{d(t-i \tau)}\right. \\
& \left.-\lambda^{2} \frac{(t+i \tau)}{(t-i \tau)}\right] .
\end{aligned}
$$

Equation (26) can be converted, using Eq. (20) as before, into a sum over Hermite-Gaussians centered on $x-p t / m$.

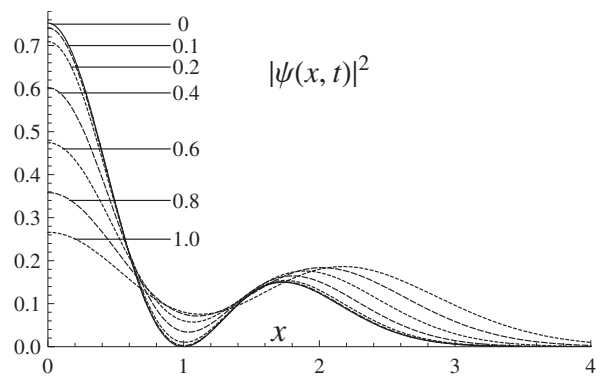

Fig. 1. The probability distribution $|\psi(x, t)|^{2}$ of the wave packet in Eq. (27), whose initial probability distribution is shown by the solid curve. Only half of the packet is shown because it remains symmetric. The probability is shown before the asymptotic regime for times $t$ $=0,0.1,0.2,0.4,0.6,0.8,1.0$ in units of $\tau$. The distance $x$ is in units of $(\hbar \tau / m)^{1 / 2}$.

\section{EXAMPLES}

Our first example is the second spatial derivative of the simple Gaussian packet $\chi(x, t)=\kappa \exp \left(-\kappa^{2} x^{2}\right)$, where $\kappa$ $=[m / 2 i \hbar(t-i \tau)]^{1 / 2}$. The packet $\chi(x, t)$ is $\Psi(x, t)$, with $\lambda=0$ in Eq. (19) and is the same as $\psi_{0}(x, t)$ in Eq. (22). Thus, we consider $\chi_{2}(x, t) \propto \partial_{x}^{2} \chi(x, t)$ which has the form

$$
\chi_{2}(x, t)=N \kappa^{3}\left(2 \kappa^{2} x^{2}-1\right) e^{-\kappa^{2} x^{2}},
$$

where $N$ is the normalization constant $(3 \sqrt{\pi} / 32)^{-1 / 2}$. The function $\chi_{2}(x, t)$ must satisfy the free Schrödinger's equation because the spatial derivative of any solution will be a solution. Although the packet $\psi_{2}(x, t)$ in Eq. (22) does not change shape as it evolves, we will show that the similar packet $\chi_{2}(x, t)$ does change shape even though it also is smooth (infinitely differentiable) everywhere and goes to zero exponentially at large distances.

Initially, the wave function $\chi_{2}(x, t)$ is real. It is also symmetric about $x=0$ and will maintain this symmetry. As shown in Fig. 1, there is a peak centered on the origin with a lower peak on either side. We will see that as it evolves, the central peak diminishes and eventually disappears while the outer peaks move further out and spread. The initial packet has $\Delta_{x}^{2}=7 \hbar \tau / 6 m$ and $\Delta_{p}^{2}=5 \hbar m / 2 \tau$, and hence $t_{p}=\tau / 5$ and $t_{x}$ $=7 \tau / 3$. We expect that the shape of the probability distribution will not change significantly for times much less than $t_{p}$. No change can be seen in Fig. 1 until $t \approx t_{p} / 3 \approx 0.06 \tau$. The maximum rate of change is at $t \approx 0.6$ and $x=0$ when visible change starts for $\delta t \approx t_{p} / 30$.

The Fourier transform of $\chi_{2}$ gives the asymptotic form as

$$
\chi_{2}(x, t) \approx \tilde{N} \frac{x^{2}}{t^{5 / 2}} \exp \left(-\frac{m \tau x^{2}}{2 h t^{2}}\right)
$$

with $\tilde{N}=2\left(m^{5} \tau^{5} / 9 \pi \hbar^{5}\right)^{1 / 4}$; this form is expected to be valid for $t \gg t_{x}$. No error in this approximation can be seen in Fig. 2 after $t \approx 7 t_{x}$.

As a second example consider the square wave packet, with

$$
\psi(x, 0)= \begin{cases}1 / \sqrt{a}, & |x|<a / 2 \\ 0, & |x| \geqslant a / 2 .\end{cases}
$$

From Eq. (7) the momentum wave function is 


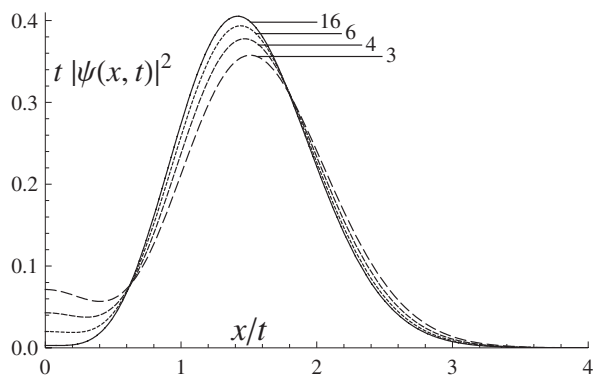

Fig. 2. The probability distribution of the same wave packet as in Fig. 1 for times $t=3,4,6,16$ when the packet is approaching its asymptotic form. In the asymptotic region $t|\psi(x, t)|^{2}$ is a function of $x / t$ only, as in Eq. (28), so we use $x / t$ as the horizontal variable, which hides the substantial spreading of these packets. For $t>7 t_{x} \approx 16 \tau$ the exact distribution is indistinguishable from the asymptotic form.

$$
\phi(p)=\sqrt{\frac{a}{2 \pi \hbar}} \frac{\sin (a p / 2 \hbar)}{a p / 2 \hbar},
$$

and therefore for $t \gg m a^{2} / \hbar$,

$$
\psi(x, t) \approx \sqrt{\frac{a m}{2 \pi i \hbar t}} \exp \left[\frac{i m x^{2}}{2 \hbar t}\right] \frac{\sin (a m x / 2 \hbar t)}{a m x / 2 \hbar t} .
$$

The exact evolution can be carried out using Eq. (2) in terms of error functions with a complex argument or Fresnel functions with a real argument. Figure 3 shows the exact probability distribution for times well before the asymptotic regime. Figure 4 shows the exact probability distribution for times when the exact distribution is approaching its asymptotic form.

In this case the initial value of $\Delta_{x}^{2}$ is $a^{2} / 12$ so that $t_{x}$ $=m a^{2} / 6 \hbar$. Figure 4 shows that the asymptotic form is a close approximation for $t>3 t_{x}$. The short-time theory in Sec. II does not apply because $\Delta_{p}=\infty$, and there are rapid changes for early times. Discontinuous wave functions are unphysical; an infinite energy would be required to produce them. Nevertheless, they can be useful as mathematically simple states which can be closely approximated by physical ones.

\section{FURTHER CONSIDERATIONS}

The discussion above is sufficiently simple and complete to be presented in an elementary course in quantum mechanics, but the following related topics might be helpful and serve as extensions for interested students.

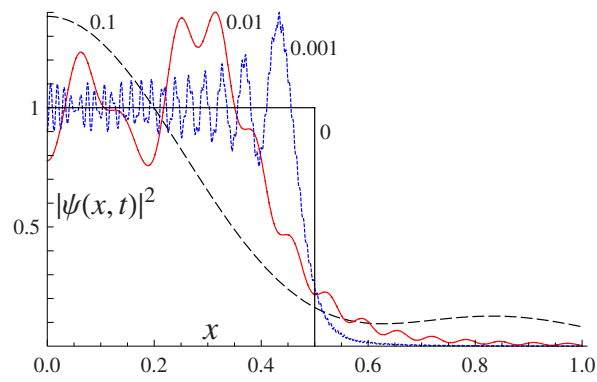

Fig. 3. The probability distribution $|\psi(x, t)|^{2}$ of the initially square wave packet in Eq. (29) for several values of the time $t=0,0.001,0.01,0.1$ for short times when the packet shows complicated behavior, but has not spread appreciably. The distance $x$ is in units of $a$, the width of the initial packet. The times are in units of $m a^{2} / \hbar$.
(1) For a more rigorous approach to the short-time behavior, we write the exact form of Eq. (5) as $\psi(x, t)$ $=\exp \left(-i \bar{p}^{2} t / 2 m \hbar\right) \psi(x-\bar{p} t / m, 0)+\delta \psi$, where

$$
\begin{aligned}
\delta \psi(x, t)= & \frac{1}{\sqrt{2 \pi \hbar}} \exp \left(\frac{-i \bar{p}^{2} t}{2 m \hbar}\right) \times \int_{-\infty}^{\infty} \exp \left[\frac{i}{\hbar} p\left(x-\frac{\bar{p} t}{m}\right)\right] \\
& \times\left(\exp \left[\frac{-i(p-\bar{p})^{2}}{2 m \hbar}\right]-1\right) \phi(p) d p .
\end{aligned}
$$

We next use the Cauchy-Schwarz inequality $\left|\int f(p) g(p) d p\right|^{2} \leqslant \int|f(p)|^{2} d p \int|(p)|^{2} d p, \quad$ with $\quad g(p)=(p$ $-\bar{p}) \phi(p)$ and $|f(p)|=\left|\sin \left[(\underline{p}-\bar{p})^{2} t / 4 m \hbar\right] /(p-\bar{p})\right|$, and the integral $\int_{-\infty}^{\infty} \sin ^{2}\left(z^{2}\right) / z^{2} d z=\sqrt{\pi}$, and obtain

$$
|\delta \psi|^{2} \leqslant \sqrt{t / \pi m \hbar^{3}} \Delta_{p}^{2}
$$

Equation (33) is a rigorous bound on the change in the wave function apart from the shift $\bar{p} t / m$. For $|\delta \psi|$ to be small compared to $\psi$ with $|\psi|^{2} \sim 1 / 2 \Delta_{x}$ (from the normalization of $\psi)$, we require $t \Delta_{p}^{4} / \pi m \hbar^{3} \ll 1 / 4 \Delta_{x}^{2}$ and hence $t \Delta_{p}^{2} / \pi m \hbar^{3}$ $\ll 1 / 4 \Delta_{x}^{2} \Delta_{p}^{2} \leqslant 1 / \hbar^{2}$ (from the uncertainty relation). That is, we require $t \ll \pi m \hbar / \Delta_{p}^{2}$, in agreement with the result in Sec. II.

(2) For a more rigorous approach to the error in the asymptotic approximation, we write the exact form of Eq. (6) as

$$
\psi(x, t)=\sqrt{\frac{m}{i t}} \exp \left[\frac{i m}{2 \hbar t}\left(x^{2}-\bar{x}^{2}\right)\right] \phi\left(\frac{m}{t}(x-\bar{x})\right)+\delta \psi,
$$

where

$$
\delta \psi=\sqrt{\frac{m}{2 \pi i \hbar t}} \exp \left[\frac{i m}{2 \hbar t}\left(x^{2}-\bar{x}^{2}\right)\right] \int_{-\infty}^{\infty} f\left(x^{\prime}\right) g\left(x^{\prime}\right) d x^{\prime},
$$

with

$$
\begin{aligned}
& f\left(x^{\prime}\right)=\exp \left[\frac{i m}{\hbar t}(\bar{x}-x) x^{\prime}\right] \frac{\exp \left[-\frac{i m}{\hbar t}\left(x^{\prime}-\bar{x}\right)^{2}\right]-1}{x^{\prime}-\bar{x}}, \\
& g\left(x^{\prime}\right)=\left(x^{\prime}-\bar{x}\right) \psi\left(x^{\prime}, 0\right) .
\end{aligned}
$$

The Cauchy-Schwarz inequality gives

$$
|\delta \psi|^{2} \leqslant \sqrt{m^{3} / \pi \hbar^{3} t^{3}} \Delta_{x}^{2} .
$$

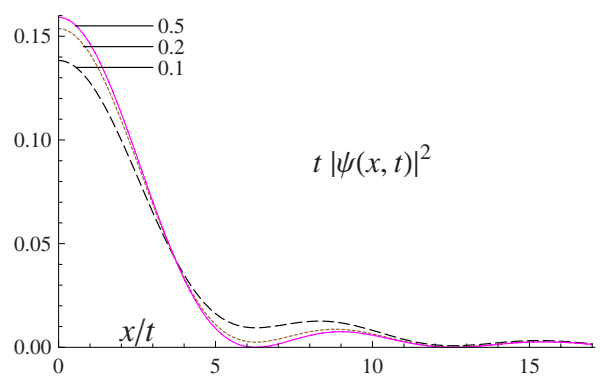

Fig. 4. The probability distribution of the initially square packet for times $t=0.1,0.2,0.5$ when the packet is approaching its asymptotic form. We show $t|\psi(x, t)|^{2}$ as a function of $x / t$. For $t \geqslant 0.5$ the exact distribution is indistinguishable from the asymptotic form. 
Equation (38) is a rigorous bound to the error in the asymptotic approximation. For $|\delta \psi|$ to be small compared to $\psi$ we require $|\delta \psi|^{2} \ll 1 / 2 \Delta_{x}$, which leads to $t \gg(4 / \pi)^{1 / 3} m \Delta_{x}^{2}$, in agreement with Sec. II.

If the wave packet has discontinuities, as in the square wave in Eq. (29), this derivation of the error is applicable only when the initial time is taken to be the instant when the discontinuities exist. At any other time $\Delta_{x}$ does not exist (because $m^{2} d_{t}^{2}\left\langle\hat{X}^{2}\right\rangle=2\left\langle\hat{P}^{2}\right\rangle$, as in Sec. IV, and $\left\langle\hat{P}^{2}\right\rangle=\infty$.) The divergence of $\left\langle\hat{x}^{2}\right\rangle$ can easily be seen for the asymptotic wave function of the initially square packet from Eq. (31).

(3) The fact that the Hermite-Gauss packets in Sec. V do not change shape is consistent with the asymptotic results in Sec. III because the Fourier transform of a Hermite-Gaussian is also a Hermite-Gaussian,

$$
\frac{1}{\sqrt{2 \pi}} \int_{-\infty}^{\infty} e^{-z^{2} / 2} H_{n}(z) e^{-i p z} d z=i^{-n} e^{-p^{2} / 2} H_{n}(p) .
$$

As observed by Lipson, ${ }^{11}$ the Fourier transform of a Hermite-Gaussian, in its role as an energy eigenfunction of the harmonic oscillator, must take the same form as a function of $p$ because the Hamiltonian is symmetric under the interchange of $p$ and $x$ (apart from constant factors).

Another way to obtain the Hermite-Gauss family of solutions is to first create $\psi_{0}(x, t)$ as in Eq. (22) as an eigenfunction of the invariant operator ${ }^{12,13} \hat{a}=\hat{x}-(t-i \tau) \hat{p} / m$ and then to repeatedly apply $\hat{a}^{\dagger}$ to obtain the solutions with $n$ $=1,2,3, \ldots$. The function $\psi_{0}(x, t)$ satisfies Schrödinger's equation because all eigenfunctions of invariant operators must do so (apart from a possible time-dependent factor) and the others do because the result of applying an invariant operator to any solution of Schrödinger's equation will also be a solution.

(4) The spatial derivative of any solution of the free Schrödinger equation is also a solution. We start with the simple Gaussian packet $\chi=\kappa \exp \left(-\kappa^{2} x^{2}\right)$, where $\kappa$ $=[m / 2 i \hbar(t-i \tau)]^{1 / 2}$, as in the first example in Sec. VI. Taking successive derivatives of $\chi$ gives a sequence of packets that also have the form of a Hermite polynomial multiplied by the original Gaussian and by a phase factor, but in this case the probability distribution changes shape as it evolves.

If we define $\chi_{n}=\partial_{x}^{n} \chi$ and use Rodrigues' equation

$$
(d / d \xi)^{n} \exp \left(-\xi^{2}\right)=(-1)^{n} H_{n}(\xi) \exp \left(-\xi^{2}\right),
$$

which is equivalent to the generating equation Eq. (20), we obtain

$$
\chi_{n} \propto \kappa^{n+1} e^{-\kappa^{2} x^{2}} H_{n}(\kappa x) .
$$

The Fourier transform of this combination of a Gaussian and an exponential is different from that in Eq. (39),

$$
\frac{1}{\sqrt{2 \pi}} \int_{-\infty}^{\infty} e^{-z^{2}} H_{n}(z) e^{-i p z} d z=\frac{(-i)^{n}}{\sqrt{2}} e^{-p^{2} / 4} p^{n} .
$$

The momentum distribution of $\chi_{n}$ is not a HermiteGaussian, and therefore these packets change shape as they evolve. The first example in Sec. VII illustrates the case $n=2$.

\section{CONCLUSION}

To gain insight into the nature of the free evolution of a wave packet, it helps to extend the evolution to the distant past as well as forward. The centroid $\langle\hat{x}\rangle$ of the packet moves with constant speed $\langle\hat{p}\rangle / m$, and we know from Sec. IV that every packet will contract and then expand, so that $\Delta_{x}$ will take its minimum value $\Delta_{\min }$ at some time $t_{\min }$. From Eq. (3) $t_{h}=m \Delta_{\min } / \Delta_{p}$ gives the time scale for the onset of linear dependence of $\Delta_{x}$ on the time; that is, for $\left|t-t_{\min }\right| \gg t_{h}, \Delta_{x}$ $\propto t$. We also know from Sec. III that for $\left|t-t_{\min }\right| \gg t_{x}$ $=2 m \Delta_{\min }^{2} / \hbar$ the shape of the wave packet will not change, but the scale will change linearly with time (consistent with the linear change in $\Delta_{x}$ ). We also found the time scale $t_{p}$ $=m \hbar / 2 \Delta_{p}^{2}$ for short-term changes in the wave function (and hence $t_{h}$ is the harmonic mean of $t_{x}$ and $t_{p}$ ). Therefore, changes in shape (other than in scale) can occur only in the waist region around $t_{\min }$ and such changes cannot be rapid over times of the order of $t_{p}$. Even in this waist region, there may be no change in shape, as exemplified by the HermiteGauss packets.

In more than one dimension the time of minimum spread and the rate of spreading may be different in each dimension, but our results apply to each dimension.

${ }^{a)}$ Electronic mail: mark.andrews@anu.edu.au

${ }^{1}$ Eugen Merzbacher, Quantum Mechanics, 2nd ed. (Wiley \& Sons, New York, 1970), p. 163, Eq. 8.91.

${ }^{2}$ We use the term wave packet to indicate that the wave function is sufficiently confined in space for $\left\langle\hat{x}^{2}\right\rangle$ to exist.

${ }^{3}$ Katsunori Mita, "Dispersion of non-Gaussian free particle wave packets," Am. J. Phys. 75, 950-953 (2007).

${ }^{4}$ Richard W. Robinett, Quantum Mechanics (Oxford University Press, New York, 1997), Eq. (3.17).

${ }^{5}$ Hans C. Ohanian, Principles of Quantum Mechanics (Prentice Hall, Englewood Cliffs, NJ, 1990), Sec. 2.4.

${ }^{6}$ See, for example, M. Daumer, D. Durr, and S. Goldstein, "On the quantum probability flux through surfaces,” J. Stat. Phys. 88, 967-977 (1997), Eq. (6), and papers referenced therein.

${ }^{7}$ Reference 1, p. 170 , Ch. 8, Prob. 8.

${ }^{8}$ Claude Cohen-Tannoudji, Bernard Diu, and Franck Lalöe, Quantum Mechanics (Wiley \& Sons, Paris, 1977), Vol. 1, Complement $\mathrm{L}_{111}$, Exer. 4.

${ }^{9}$ Eugen Merzbacher, Quantum Mechanics, 3rd ed. (Wiley \& Sons, New York, 1998), p. 75.

${ }^{10}$ Reference 1, p. 161, Eq. (8.78)

${ }^{11}$ S. G. Lipson, "Self-Fourier objects and other self-transform objects: Comment," J. Opt. Soc. Am. A 10, 2088-2089 (1993).

${ }^{12}$ Mark Andrews, "Total time derivatives of operators in elementary quantum mechanics," Am. J. Phys. 71, 326-332 (2003).

${ }^{13}$ Mark Andrews, "Invariant operators for quadratic Hamiltonians," Am. J. Phys. 67, 336-343 (1999). 